\title{
Autophagy in stromal fibroblasts promotes tumor desmoplasia and mammary tumorigenesis
}

\author{
Jenny A. Rudnick, ${ }^{1,6}$ Teresa Monkkonen, ${ }^{1,6}$ Florie A. Mar, ${ }^{1,2}$ James M. Barnes, ${ }^{3}$ Hanna Starobinets, ${ }^{1,2}$ \\ Juliet Goldsmith, ${ }^{1,2}$ Srirupa Roy, ${ }^{1}$ Sofía Bustamante Eguiguren, ${ }^{1}$ Valerie M. Weaver, ${ }^{3,4,5}$ \\ and Jayanta Debnath ${ }^{1}$ \\ ${ }^{1}$ Department of Pathology, Helen Diller Family Comprehensive Cancer Center, University of California at San Francisco, \\ San Francisco, California 94143, USA; ${ }^{2}$ Biomedical Sciences Graduate Program, University of California at San Francisco, \\ San Francisco, California 94143, USA; ${ }^{3}$ Department of Surgery, Center for Bioengineering and Tissue Regeneration, University \\ of California at San Francisco, San Francisco, California 94143, USA; ${ }^{4}$ Department of Anatomy, University of California \\ at San Francisco, San Francisco, California 94143, USA; ${ }^{5}$ Department of Bioengineering and Therapeutic Sciences, University \\ of California at San Francisco, San Francisco, California 94143, USA
}

Autophagy inhibitors are currently being evaluated in clinical trials for the treatment of diverse cancers, largely due to their ability to impede tumor cell survival and metabolic adaptation. More recently, there is growing interest in whether and how modulating autophagy in the host stroma influences tumorigenesis. Fibroblasts play prominent roles in cancer initiation and progression, including depositing type 1 collagen and other extracellular matrix (ECM) components, thereby stiffening the surrounding tissue to enhance tumor cell proliferation and survival, as well as secreting cytokines that modulate angiogenesis and the immune microenvironment. This constellation of phenotypes, pathologically termed desmoplasia, heralds poor prognosis and reduces patient survival. Using mouse mammary cancer models and syngeneic transplantation assays, we demonstrate that genetic ablation of stromal fibroblast autophagy significantly impedes fundamental elements of the stromal desmoplastic response, including collagen and proinflammatory cytokine secretion, extracellular matrix stiffening, and neoangiogenesis. As a result, autophagy in stromal fibroblasts is required for mammary tumor growth in vivo, even when the cancer cells themselves remain autophagy-competent. We propose the efficacy of autophagy inhibition is shaped by this ability of host stromal fibroblast autophagy to support tumor desmoplasia.

[Keywords: cancer-associated fibroblasts; autophagy; tumor microenvironment; collagen; breast cancer]

Supplemental material is available for this article.

Received October 10, 2020; revised version accepted May 24, 2021.

Macroautophagy, referred to here as autophagy, is a cellular degradation and recycling pathway that promotes tumor cell survival and resistance to therapy (Amaravadi et al. 2019); as a result, autophagy inhibition using antimalarials is in clinical trials for several cancers, including breast. Emerging evidence suggests that inhibiting autophagy in the host stroma negatively impacts tumor growth. Notably, studies in Drosophila demonstrate that the systemic induction of autophagy in host tissues promotes the aggressive growth and invasion of $\mathrm{RasV} \mathrm{scrib}^{-/-}$tumors throughout the fly (Katheder et al. 2017). In mouse models of pancreatic cancer, autophagy is required for pancreatic stellate cells, a key constituent of the tumor stroma, to produce and secrete the nonessential amino

\footnotetext{
${ }^{6}$ These authors contributed equally to this work.

Corresponding author: jayanta.debnath@ucsf.edu

Article published online ahead of print. Article and publication date are online at http://www.genesdev.org/cgi/doi/10.1101/gad.345629.120.
}

acid alanine, which is used for the growth and survival of pancreatic tumor cells in adverse microenvironments (Sousa et al. 2016). Furthermore, whole-body deletion of Atg7 or Atg5 reduces the systemic levels of circulating arginine, which impairs the growth of certain tumors that are arginine auxotrophs (Poillet-Perez et al. 2018). In these models, degradation via autophagy in host stromal cells is proposed to elaborate key metabolites, namely, amino acids, which can sustain the core metabolic functions of the proliferating tumor compartment. In addition to hosttumor metabolite exchange, autophagy promotes the cellular secretion of diverse proteins (Cadwell and Debnath 2018; Monkkonen and Debnath 2018). However, it

(C) 2021 Rudnick et al. This article is distributed exclusively by Cold Spring Harbor Laboratory Press for the first six months after the full-issue publication date (see http://genesdev.cshlp.org/site/misc/terms.xhtml). After six months, it is available under a Creative Commons License (Attribution-NonCommercial 4.0 International), as described at http://creativecommons.org/licenses/by-nc/4.0/. 
remains unclear whether and how these autophagy-regulated secretory events exert non-cell-autonomous control on the tumor microenvironment.

The fibroblasts residing within solid tumors, termed cancer-associated fibroblasts (CAFs), represent principal components of the stroma that modulate tumor cell proliferation and behavior through diverse mechanisms, including the synthesis of growth and angiogenic factors, cytokines, extracellular matrix (ECM) components, and proteases. In vitro coculture of tumor cells with CAFs, or coinjection in vivo, is frequently sufficient to augment tumor cell proliferation and invasiveness (LeBleu and Kalluri 2018). Moreover, many solid tumors exhibit striking histological evidence of fibroblast activation, increased tissue stiffness, and inflammation, phenotypes that are collectively termed desmoplasia and herald poor prognosis in diverse human cancers (Schedin and Keely 2011; LeBleu and Kalluri 2018).

Nevertheless, much remains to be learned about the cellular mechanisms that generate and maintain this desmoplastic fibrotic response. Intriguingly, autophagy is upregulated in fibroblasts in multiple models. In a breast cancer model, tumor-dependent hypoxic stress increased CAF autophagy to promote cancer cell survival (Martinez-Outschoorn et al. 2010). Recent work in head and neck cancer models correlated fibroblast autophagy with poor patient outcome, which was associated with increased cytokine secretion (New et al. 2017). These studies also found that treatment with autophagy inhibitors in vivo enhanced cisplatin efficacy in immunocompromised mice bearing xenografted tumors (New et al. 2017).

Based on these findings, we scrutinized whether autophagy in host stromal fibroblasts contributes to desmoplasia during tumor progression. We leveraged autochthonous tumor models and syngeneic transplantation to genetically ablate fibroblast autophagy, and interrogate how polyoma middle $\mathrm{T}$ antigen-driven (PyMT) mammary tumorigenesis is impacted in vivo. In all of these models, loss of fibroblast autophagy reduced tumor progression. Furthermore, we uncovered that autophagy in fibroblasts promotes collagen secretion, which supports a stiff desmoplastic stroma permissive for mammary tumor growth in vivo.

\section{Results}

FSP-Cre-mediated autophagy deletion impairs polyoma middle T-driven mammary tumorigenesis

As autophagy promotes proinflammatory cytokine (Narita 2011; Deretic et al. 2013; Lock et al. 2014) and collagen (Cinque et al. 2015; Forrester et al. 2019) secretion, we hypothesized that autophagy may enable protumorigenic functions of fibroblasts (Erez et al. 2010; LeBleu and Kalluri 2018). To scrutinize how genetic ablation of autophagy in fibroblasts impacts mammary tumorigenesis in vivo, we generated transgenic mice bearing conditional alleles independently targeting two essential Atgs (Atg12 or Atg5) using Cre recombinase under the fibroblast-specific protein promoter (FSP-Cre) and PyMT antigen expression driven by the mouse mammary tumor virus promoter (MMTV-PyMT) (Lin et al. 2003; Bhowmick et al. 2004; Kuma et al. 2004; Malhotra et al. 2015). These mice $\left(\right.$ Atg $12^{f l / f l_{i} \mathrm{Cre}+} ;$ PyMT + and Atg $5^{\text {fl/fl; Cre+;} P y M T+)}$ carried a lox-stop-lox RFP (red fluorescent protein) cassette at the Rosa26 locus (Luche et al. 2007). Mice were born at Mendelian ratios with no obvious viability defects. Sorting the RFP+ fraction from digested mammary glands of $\operatorname{Atg} 5^{f l / f l ; C r e+}$ and $\operatorname{Atg} 12^{f l / f l ; C r e+}$ mice confirmed effective recombination in vivo, which was restricted to a nonepithelial $\left(\mathrm{EpCAM}^{-}\right.$) subpopulation (Supplemental Fig. S1).

We monitored mammary tumor development in these autochthonous models over $120 \mathrm{~d}$. At the end point, the autophagy-competent cohort (FSP-Cre negative; $\operatorname{Atg} 5^{f l / f l ; C r e-} ;$ PyMT+ and Atg12 ${ }^{f l / f l_{;} C r e-} ;$ PyMT+l exhibited larger tumors (Fig. 1A,B) relative to FSP-Cre;Atg ${ }^{f l / f 1}$; $P y M T+$ mice at the end stage. The time to palpable tumor formation was not altered in FSP-Cre;Atg5/12 fl/fl mutants, although the percentage of mice with palpable tumors was reduced at $85 \mathrm{~d}$ (Fig. 1C-E). Stromal autophagy ablation in the Atg $5^{f l / 1 ; C r e+;}$ PyMT+ and Atg $12^{f l / f l ; C r e+} ;$ PyMT+ tumors was confirmed by increased p62/sequestosome 1 protein staining in the stroma compared with controls, with no obvious changes in aSMA staining (Fig. 1F).

\section{Autophagy deletion in stromal fibroblasts impairs mammary tumorigenesis in syngeneic transplantation models}

Close inspection of Atg $5^{\mathrm{fl} / 1 ; \mathrm{Cre+}}$;PyMT+ and Atg12 ${ }^{\mathrm{fl} / \mathrm{fl} ; \mathrm{Cre+}}$; PyMT + tumors revealed p62-positive cells within the tumor compartment (Fig. 1F), which was likely due to FSP-Cre-mediated autophagy deletion in nonfibroblast cell types, such as $\mathrm{CD} 45^{+}$cells (Bhowmick et al. 2004; Fischer et al. 2015). Thus, we adopted transplantation assays to more precisely scrutinize stromal fibroblast autophagy in tumor progression. We first isolated primary mouse mammary fibroblasts (MMFs) from conditional Atg12 or Atg5 RFP+ mice $\left(\right.$ Atg12 $12^{f l / f 1}$ or $\left.A \operatorname{tg} 5^{f l / f 1}\right)$ or wild-type (WT) $\mathrm{RFP}+$ mice $\left(R F P^{+/+; A d C r e}\right)$. To ablate autophagy, MMFs were infected with adenovirus-driven Cre recombinase (AdCre). Immunoblotting confirmed loss of lipidated LC3 (LC3-II) and p62/SQSTM1 accumulation, confirming AdCre rendered both $A \operatorname{tg} 12^{f l / f 1}$ and $A \operatorname{tg} 5^{f l / f 1}$ MMFs autophagy-deficient (Fig. 2A; Supplemental Fig. S2A). We verified our isolation techniques by immunostaining MMFs for aSMA and vimentin expression, along with the lack of expression of mammary epithelial cytokeratins CK8 and CK14 (Supplemental Fig. S2B).

Because autophagy is required for the differentiation of hepatic stellate cells into myofibroblasts in vitro and in vivo (Thoen et al. 2011; Hernández-Gea et al. 2012), we assessed whether autophagy deficiency in mammary fibroblasts influenced fibroblast activation. In contrast to stellate cells, ablation of autophagy in MMFs did not prevent expression of established fibroblast differentiation markers in vitro (Rudnick and Kuperwasser 2012) including FAP, FSP1, aSMA, and fibronectin (FN1) (Fig. 2B); the coordinate reduction in these activation markers was not observed (Supplemental Fig. S2C). We also tested whether 
A

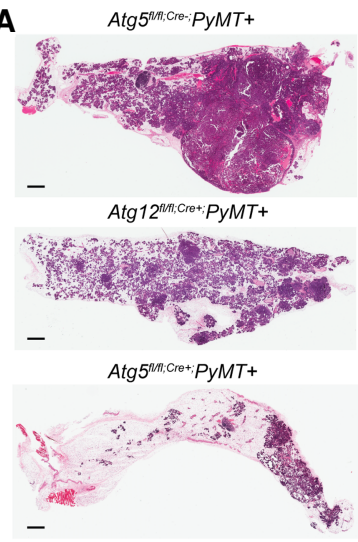

E

\begin{tabular}{|c|c|c|c|}
\hline Cohort & \# Mice in Cohort & \% Palpable 85 days & Final Tumor Vol. \\
Atg5 and Atg12 $2^{f f f t ;}$ Cre-; PyMT+ & 24 & 25 & $462.5 \pm 154.7$ \\
\hline Atg12 $2^{f f f t ;}$ Cret; PyMT+ & 17 & 11.8 & $115.0 \pm 38.0$ \\
\hline Atg5 $5^{f f f ;} ;$ Cre+; PyMT+ & 8 & 0 & $16.00 \pm 7.4$ \\
\hline
\end{tabular}
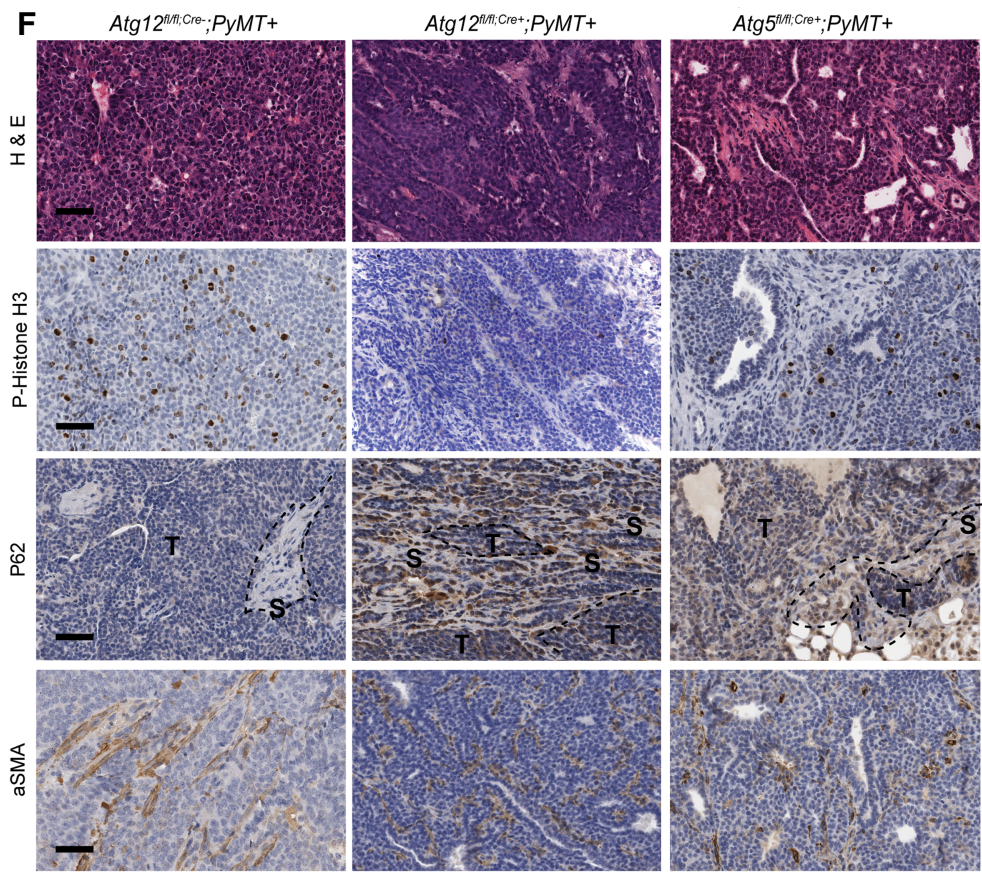

B

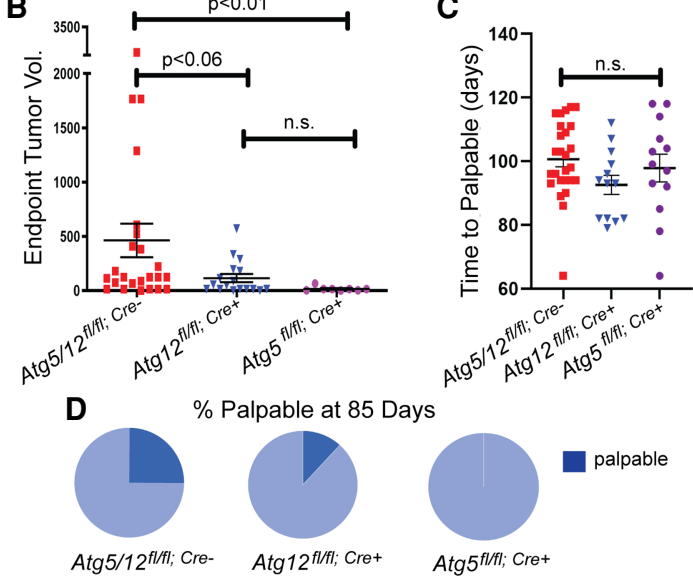

PyMT+

Figure 1. FSP-Cre mediated stromal fibroblast autophagy ablation impedes autochthonous PyMT mammary tumor growth. $(A)$ $\mathrm{H} \& \mathrm{E}$ images of representative fourth inguinal mammary glands from FSP-Cre;PyMT+ mice at $108 \mathrm{~d}$ displaying mammary hyperplasia. Scale bar, $1.5 \mathrm{~mm}$. (B) Scatter plot of end point tumor volume (in cubic millimeters) for FSPCre;PyMT+ mice. Mean \pm SEM. FSP-Cre negative, $n=24$ mice; Atg12 ${ }^{f l / f l ; C r e+}{ }_{;} P y M T+$, $n=17$ mice; and $\operatorname{Atg} 5^{\text {fl/fl; } \mathrm{Cre+}} ;$ PyMT+, $n=8$ mice. End point determined by mice in the control cohort requiring euthanasia. One way nonparametric ANOVA. (C) Scatter plot of age at first detection of palpable tumor. Control, $n=26$; FSP-Cre; Atg12 ${ }^{f l / f 1}, n=13$; and FSP-Cre;Atg5 $5^{f 1 / f 1}, n=13$; ANOVA. (D) Percentage palpable at $85 \mathrm{~d}$, referring to the percentage of mice with tumors $\geq 3 \mathrm{~mm}$ in diameter (dark blue). (E) Summary of tumor data from FSP-Cre;PyMT+ mice. $(F)$ Representative $\mathrm{H} \& \mathrm{E}$, phosphorylated histone $\mathrm{H} 3$ (pH3), p62, and aSMA immunohistochemistry of end stage FSP-Cre;PyMT+ tumors. Scale bar, $50 \mu \mathrm{m}$. (T) Region with tumor cells, (S) stromal region enriched for fibroblasts.

the $\mathrm{TGF}^{\beta}$ signaling axis critical for CAF activation (Rønnov-Jessen and Petersen 1993; Kojima et al. 2010) was inhibited upon autophagy deletion. TGF ${ }^{\beta}$-mediated fibroblast activation was not impaired upon genetic autophagy deletion; baseline total SMAD2/3 levels and SMAD2/3 phosphorylation upon $\mathrm{TGF}^{\beta}$ treatment appeared intact or increased in Atg5/12-deficient MMFs (Fig. 2C; Supplemental Fig. S3A-C). Collectively, these data indicate autophagy deletion does not broadly impair MMF differentiation or activation in vitro. To determine how ablation of autophagy influenced cellular bioenergetics in autophagy-deficient MMFs, we evaluated intracellular ATP levels, which were decreased upon loss of autophagy (Fig. 2D). Upon treatment with the mitochon- drial ATP synthase inhibitor oligomycin, no differences in autophagy-deficient MMFs relative to controls were observed (Fig. 2D). Finally, Ki67 staining confirmed Atg5/ 12-deficient MMFs displayed reduced proliferation in vitro relative to controls (Fig. 2E), consistent with previous studies (Liao et al. 2019).

Next, we tested whether fibroblast autophagy deficiency influenced mammary tumorigenesis in transplantation models. Given the largely concordant phenotypes with Atg5/12 deletion in fibroblasts in vitro and in vivo, we focused on Atg12-deficient MMFs to streamline our in vivo studies. First, we coinjected autophagy-competent $\left(\mathrm{RFP}^{+/+ \text {AdCre }}\right)$ or autophagy-deficient $\left(\right.$ Atg12 $\left.{ }^{\text {fl/fl; AdCre }}\right)$ MMFs with autophagy-competent PyMT tumor cells 


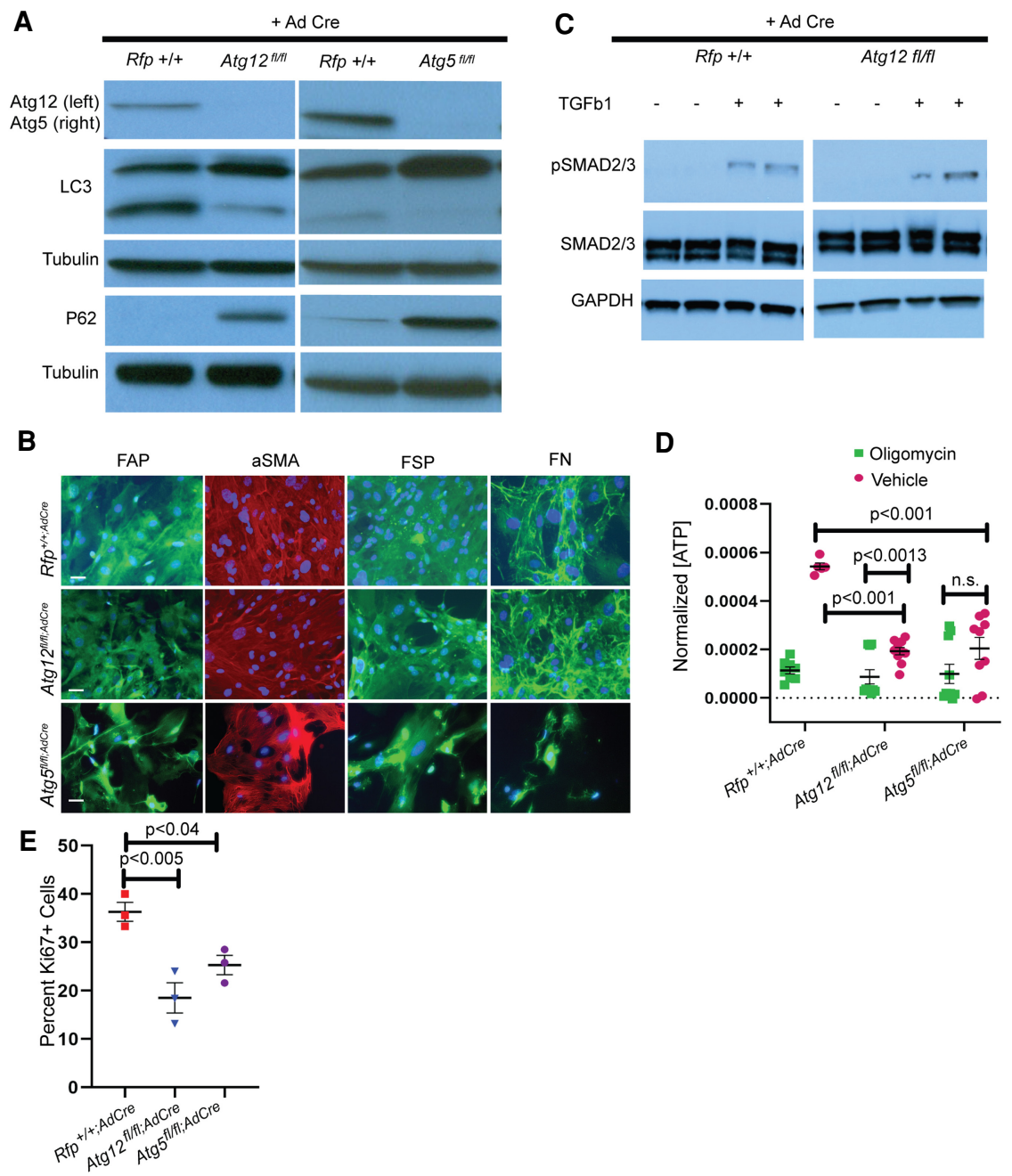

Figure 2. Autophagy deficiency does not impair activation of mouse mammary fibroblasts (MMFs). (A) Immunoblot of $\mathrm{RFP}^{+/+ \text {AdCre }}$ Atg12 $2^{\text {fl/fl; AdCre, and }}$ Atg $5^{f l / f 1 ; A d C r e}$ MMF lysates confirming Cremediated deletion of Atg12 or Atg5 and autophagy deficiency (loss of LC3-II and accumulation of p62/SQSTM1). Tubulin is the loading control. $(B)$ Representative immunofluorescence staining of fibroblast activation markers in $R F P^{+/+; A d C r e}$, Atg12 1 fl/fl;AdCre, and Atg5 $5^{f l / f 1 ; A d C r e}$ MMFs grown on type 1 collagen. Scale bar, 100 $\mu \mathrm{m}$. (C) Immunoblot of phospho- and total SMAD $2 / 3$ levels in $R F P^{+/+; A d C r e}$ and Atg12 $2^{f l / f 1 ; A d C r e}$ MMFs grown on type 1 collagen with and without recombinant $\mathrm{TGF}^{\beta} 1$. $(D)$ Intracellular ATP concentrations of autophagy-competent $\left(R F P^{+/+; A d C r e}\right)$ or -deficient $\left(\operatorname{Atg} 12^{f l / f 1 ; A d C r e}\right.$ and Atg $\left.5^{f l / f l ; A d C r e}\right)$ MMFs treated with $5 \mu \mathrm{M}$ oligomycin (green squares) or vehicle control (red circles) for $3 \mathrm{~h}$. Concentrations normalized to final cell count. Mean \pm SEM from three independent experiments; $R F P^{+/+ \text {AdCre }}$, vehicle, $n=5$; $R F P^{+/+; A d C r e}$, oligomycin, $n=8$; Atg12 $2^{f l / f 1 ; A d C r e}$, vehicle, $n=10 ; \operatorname{Atg} 12^{A / f / A d C r e}$, oligomycin, $n=$ 10; Atg5 $5^{\text {fl/fl; AdCre }}$, vehicle, $n=9 ;$ Atg $5^{\text {fl/fl; AdCre }}$, oligomycin, $n=10$. Two-way ANOVA, Sidak's multiple comparisons test. (E) Ki67 immunocytochemistry of control and Atg5/ $12^{\text {fl/fl; AdCre }}$ MMFs. Each data point represents percentages from $>75$ cells. ANOVA. into mammary glands of syngeneic $\mathrm{C} 57 \mathrm{Bl} / 6$ recipient mice. Mice implanted with a comixture of PyMT cells and autophagy-deficient MMFs (Atg12 $1 / f 1$;AdCre comix) had significantly reduced tumor volume at end stage compared with the $R \mathrm{FP}^{+/+; A d C r e}$ comix or mice injected with PyMT tumor cells only but no difference in tumor latency (Fig. 3A-C). These results corroborated our findings from the autochthonous FSP-Cre-driven autophagy deletion models and suggested that reduced PyMT tumor burden in FSP-Cre-driven autophagy deficiency is likely due to the specific ablation of autophagy in fibroblasts.

\section{Autophagy deficiency in fibroblasts disrupts collagen architecture}

To extend these results, we used an established technique in which the mammary gland is cleared at $3 \mathrm{wk}$ of age, leaving behind a fat pad devoid of epithelium (Deome et al. 1959). Stromal fibroblasts were implanted into the mammary fat pad (MFP) and allowed to engraft for 3 wk (Kuperwasser et al. 2004). Upon introducing Atg12 $2^{f l / f 1 ; A d C r e}$ or $R F P^{+/+ \text {AdCre }}$ MMFs into the cleared MFPs of host mice (Fig. 4A), the primed MFPs showed comparable numbers of RFP+ MMFs, indicating fibroblast engraftment in vivo was not impaired upon autophagy ablation (Fig. 4B). Immunohistochemistry confirmed RFPpositive MMFs in primed MFPs (Fig. 4C). Intriguingly, both Masson's trichrome and Picrosirius red staining of the primed glands at 3 wk demonstrated aberrant collagen architecture and significantly reduced collagen deposition in the MFPs primed with autophagy-deficient MMFs (Fig. 4D,E). Trichrome staining of FSP-Cre;Atg12 fl/fl tumors also showed reduced collagen deposition relative to controls (Fig. 4F).

ECM stiffening and enhanced collagen deposition are hallmarks of aggressive cancer and promote signaling associated with tumor growth and invasion (Schedin and Keely 2011). Given the reduced collagen content in these autophagy-deficient tissues, we tested whether the MFPs primed with autophagy-deficient MMFs supported mammary cancer growth in vivo. Three weeks following MMF engraftment, autophagy-competent PyMT tumor cells were implanted into the primed MFPs (Fig. 5A). Mice primed with autophagy-deficient MMFs showed significantly impaired PyMT tumor volume at end point and improved survival compared with controls (Fig. 5B). In fact, a 
A

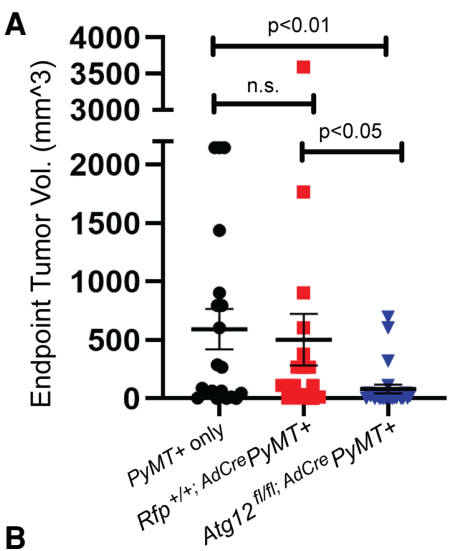

\begin{tabular}{|r|c|c|c|}
\hline & \# Mice in Cohort & Time to Palpable & Final Tumor Vol. \\
\hline Rfp $^{+/+;}$AdCre $P y M T+$ & 17 & $31.7+/-2.7$ days & $618+/-202 \mathrm{~mm}^{\wedge} 3$ \\
\hline${\text { Atg } 12^{\text {fl/f; } ; \text { AdCre }} \text { PyMT+ }}$ & 21 & $34.5+/-2.6$ days & $194+/-95 \mathrm{~mm}^{\wedge} 3$ \\
\hline
\end{tabular}

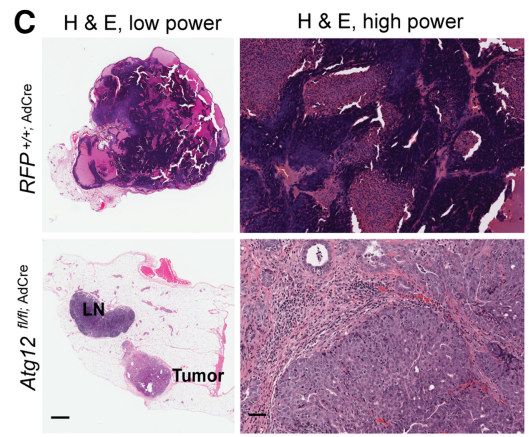

Figure 3. Autophagy deletion in stromal fibroblasts impairs PyMT tumorigenesis in syngeneic cotransplantation models. $(A)$ Orthotopic injection of primary PyMT tumor cells comixed with autophagy-competent $\left(R F P^{+/+A d C r e}\right)$ or -deficient $\left(\right.$ Atg $\left.12^{f l / f f_{;} \text {AdCre }}\right)$ MMFs or injected alone (РуMT only) into fourth inguinal mammary glands of 6- to 8wk-old C57B/6 females. Scatter plot showing end point tumor volume $\left(\mathrm{mm}^{3}\right)$ for comixed tumors. Data from three independent experiments. End point based on mice in the control $\left(R F P^{+/+; A d C r e}\right.$ comix) cohort requiring euthanasia. Mean \pm SEM from three independent experiments. PyMT only $n=20 ; R F P^{+/+; A d C r e}$ comix, $n=22 ;$ Atg12 $2^{f l / f 1 ; A d C r e}$ comix, $n=26$. Nonparametric ANOVA. (B) Summary of tumor data from comix experiments. (C) Representative low-power (scale bar, $1.5 \mathrm{~mm}$ ) and high-power (scale bar, $100 \mu \mathrm{m}) \mathrm{H} \& \mathrm{E}$ images of comix tumors from data shown in $A$. (LN) Lymph node. subset of mice primed with autophagy-competent MMFs had prominent tumor burden requiring euthanasia as early as $50 \mathrm{~d}$, whereas mice primed with autophagy-deficient MMFs failed to reach end stage tumor burden by the termination of the study (Fig. 5B). Consistent with the in vitro fibroblast activation marker analysis, SMA and FSP positivity was similar between tumors primed with autophagy-competent versus autophagy-deficient MMFs (Fig. 5D). These data further support that autophagy deficiency in stromal fibroblasts suppresses mammary tumorigenesis, even when tumor cells are autophagy competent.

\section{Effects of fibroblast autophagy on cytokine secretion}

Various reports demonstrate autophagy deficiency results in impaired cytokine secretion (Narita 2011; Deretic et al. 2013; Lock et al. 2014; New et al. 2017), which may modulate the tumor microenvironment permissive for tumor growth. Hence, we performed a mouse cytokine array (Supplemental Tables S1, S2; Supplemental Fig. S4) to broadly view cytokine signaling upon autophagy deletion in fibroblasts. Similar to previous studies (Narita 2011; Lock et al. 2014; New et al. 2017), secreted IL-6 was significantly reduced in the CM of both Atg5/12 $2^{f / / f i \text { AdCre }}$ MMFs compared with controls (Fig. 5E), while the steady-state protein levels of IL-6 levels were unaltered, suggesting impaired secretion by autophagy-deficient fibroblasts (Fig. 5F; Supplemental Fig. S3). IL-6 acts as a pleiotropic cytokine that instructs both inflammatory and profibrotic phenotypes associated with a protumorigenic microenvironment, and promotes neoangiogenesis in solid tumors (Bunt et al. 2007; Bromberg and Wang 2009; Fielding et al. 2014). Remarkably, upon evaluating microvessel density (MVD), we observed significantly reduced neoangiogenesis in the primed regions with $\operatorname{Atg} 12^{\text {fllffl AdCre }}$ MMFs (Fig. 5G). To further scrutinize the IL-6 pathway in vivo, we evaluated serum IL-6 levels of FSP-Cre;Atg5/ $12^{f l / f l}$ and control $\mathrm{Cre}-$ mice, but found no significant dif- ferences between these cohorts (Supplemental Fig. S5A). We also assayed for pSTAT3 (Y705), a downstream marker of IL-6 activation, in the comix or prime tumors with Atg12 ${ }^{f l / f 1}$ MMFs. However, few pSTAT3-positive cells were detected in either tumor or stromal cells, with no obvious differences between $\operatorname{Atg} 12^{f l / f 1}$ MMFs versus controls (Supplemental Fig. S5B). Overall, despite reduced neoangiogenesis in stroma derived from autophagy-deficient MMFs, the lack of differences in pSTAT3 signaling or serum IL-6 levels in vivo suggest that decreased IL-6 secretion due to fibroblast autophagy inhibition is unlikely to contribute to reduced tumorigenesis in these models.

Because fibroblast autophagy inhibition impacted the secretion of multiple cytokines in vitro (Supplemental Fig. S4), we further scrutinized its effects on immune cell recruitment in vivo. Immunostaining revealed similar numbers of CD45- and CD11b-positive cells in Atg12 $2^{f / f f ;}$;dCre MMF primed regions relative to controls (Supplemental Fig. S6A,B). In contrast, F4/80-, CD3-, and CD4-positive cells were all significantly decreased in Atg $12^{f l / f f ; A d C r e}$ MMF primed regions compared with controls (Supplemental Fig. S6A,B), suggesting that fibroblast autophagy influences the recruitment of certain immune cell populations in vivo.

\section{Stromal fibroblast autophagy promotes tissue stiffness}

Due to the dramatic changes in collagen deposition observed upon loss of fibroblast autophagy in the primed MFPs (Fig. 4), we scrutinized whether differences in ECM stiffness contributed to the ability of autophagy-deficient MMFs to suppress tumor growth. Atomic force microcopy (AFM) mechanical testing corroborated that MFPs primed with autophagy-deficient MMFs exhibited significantly reduced tissue stiffness compared with controls (Fig. 6A). Furthermore, collagen gel contractility of autophagy-competent and -deficient MMFs in vitro showed that autophagy depletion reduces MMF 


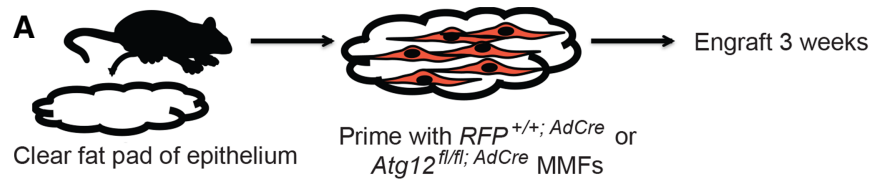

B

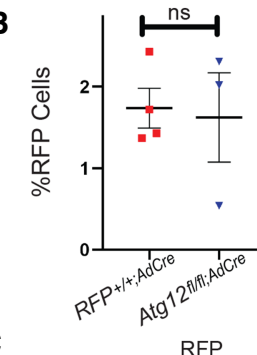

C

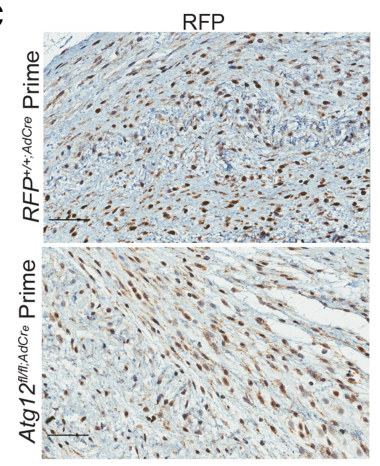

E

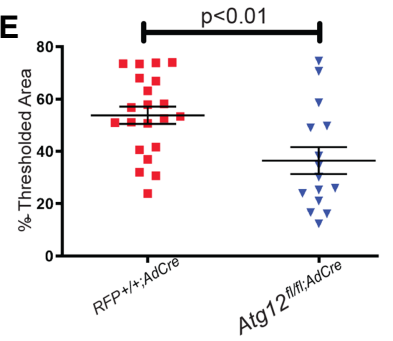

D

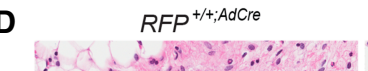

$$
\text { w }
$$
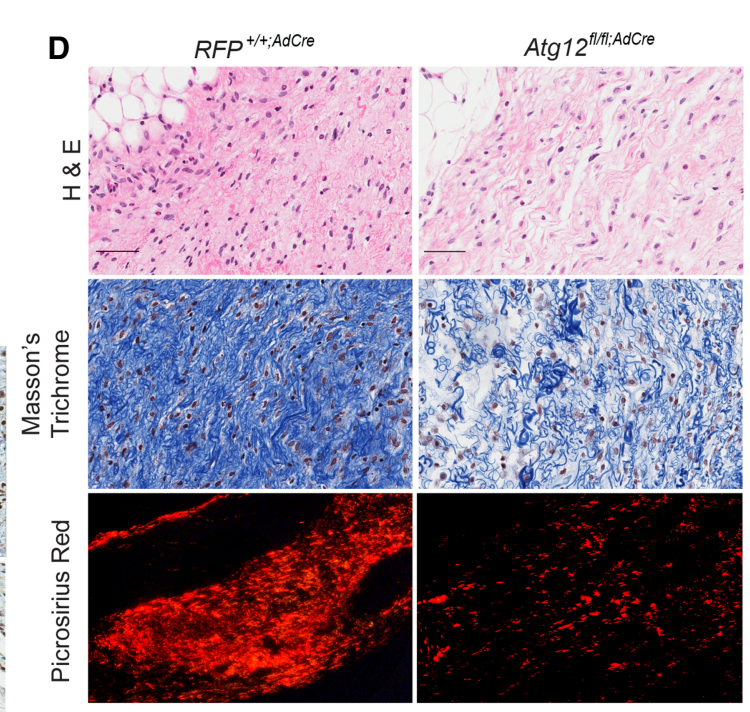

F

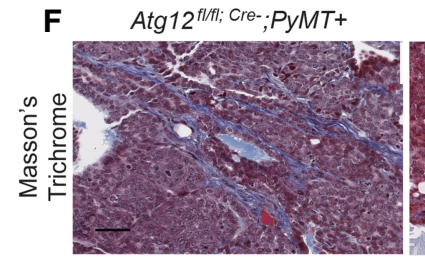

$\operatorname{Atg}^{\text {fffff; }} \mathrm{Cre+}^{\mathrm{C}}$ PyMT+

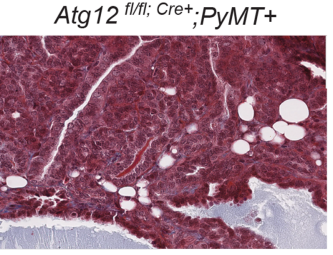

Figure 4. Mammary fat pads primed with autophagy-deficient MMFs exhibit reduced type 1 collagen deposition in vivo. (A) Priming experiment schematic. The right fourth inguinal mammary gland of 3-wk-old C57B/ 6 females cleared of mammary epithelium and primed with autophagy-competent $\left(R F P^{+/+; A d C r e}\right)$ or -deficient $\left(\right.$ Atg $\left.12^{f 1 / f 1_{;} \text {AdCre }}\right)$ MMFs. Tissues analyzed $3 \mathrm{wk}$ after engraftment. (B) FACS for percentage of RFP+ MMFs after engraftment. Mean \pm SEM. RFP $^{+/+; A d C r e}, \quad n=5$ mice; Atg $12^{f l / f 1 ; A d C r e}$, $n=3$ mice. Student's $t$-test. $(C)$ Representative IHC for RFP expression in autophagycompetent $\left(R F P^{+/+; A d C r e}\right)$ or -deficient $\left(\right.$ Atg12 $\left.{ }^{f l / f f_{;} \text {AdCre }}\right)$ primed MFPs. Scale bar, $50 \mu \mathrm{m}$. (D) Representative H\&E-stained (top), Masson's trichrome-stained (middle), and Picrosirius red-stained (bottom) MFPs primed with autophagy-competent $\left(R F P^{+/+; A d C r e}\right)$ or -deficient (Atg12 $2^{\text {fl/fi;AdCre }}$ ) MMFs. Scale bar, $50 \mu \mathrm{m}$. (E) Quantification of thresholded Picrosirius red intensity. Mean \pm SEM. $R F P^{+/+; A d C r e}$ prime, $n=15$ fields from three mice; Atg12 $2^{f l / f 1 ; A d C r e}$ prime, $n=15$ fields from three mice. Student's $t$-test. $(F)$ Masson's trichromestained $\operatorname{Atg} 12^{f l / f 1}$ and FSP-Cre;Atg12 $12^{f l / f l}$ PyMT tumors. contractility of collagen gels (Fig. 6B). Hence, autophagy functionally promotes both tissue stiffness and fibroblast contractility, two hallmarks of CAF function.

\section{Autophagy in mouse mammary fibroblasts is required for type 1 collagen secretion and procollagen proteostasis}

We hypothesized the reduced stiffness of the autophagydeficient primed MFPs may be due to impaired collagen secretion, which has been described in other autophagydeficient cell types (Cinque et al. 2015; Forrester et al. 2019). Following culture with a long-acting stable derivative of ascorbic acid to stimulate collagen production, we observed reduced acid-soluble collagen secretion by autophagy-deficient MMFs in vitro compared with controls (Fig. 6C). Acutely inhibiting autophagy in WT MMFs with Spautin-1, a potent and selective autophagy inhibitor, similarly impaired collagen secretion (Fig. 6D). There was no change in transcript levels of type 1 collagen or collagen-stabilizing lysyl oxidase genes Lox1 and Loxl2 (Supplemental Fig. S6C), suggesting the reduced extracellular collagen observed in autophagy-deficient fibroblasts was due to a collagen secretory defect. Recent work has uncovered that impaired collagen secretion with autophagy deficiency arises from intracellular defects in procollagen degradation via the lysosome. Because a significant fraction of procollagen synthesized in the endoplasmic reticulum (ER) is inefficiently folded, it is normally degraded in the lysosome via autophagy-dependent mechanisms. Hence, the genetic loss of autophagy results in impaired procollagen proteostasis, thereby impairing collagen secretion due to the loss of ER quality control (Cinque et al. 2015; Forrester et al. 2019|. Accordingly, we scrutinized whether the autophagic machinery was required for the lysosome clearance of type 1 procollagen (PC1) in MMFs using immunofluorescence staining (Forrester et al. 2019). Indeed, autophagy-competent MMFs displayed procollagen translocation to the lysosome, which was significantly enhanced upon treatment with the lysosomal acidification inhibitor bafilomycin A (Baf A), resulting in increased colocation of type 1 procollagen (PC1) with the lysosomal marker LAMP1 in Baf A-treated, autophagy-competent MMFs (Fig. 7A,F; Supplemental Fig. S7). In contrast, the lysosomal translocation of PC1 was significantly reduced in Atg5- or Atg12-deficient 


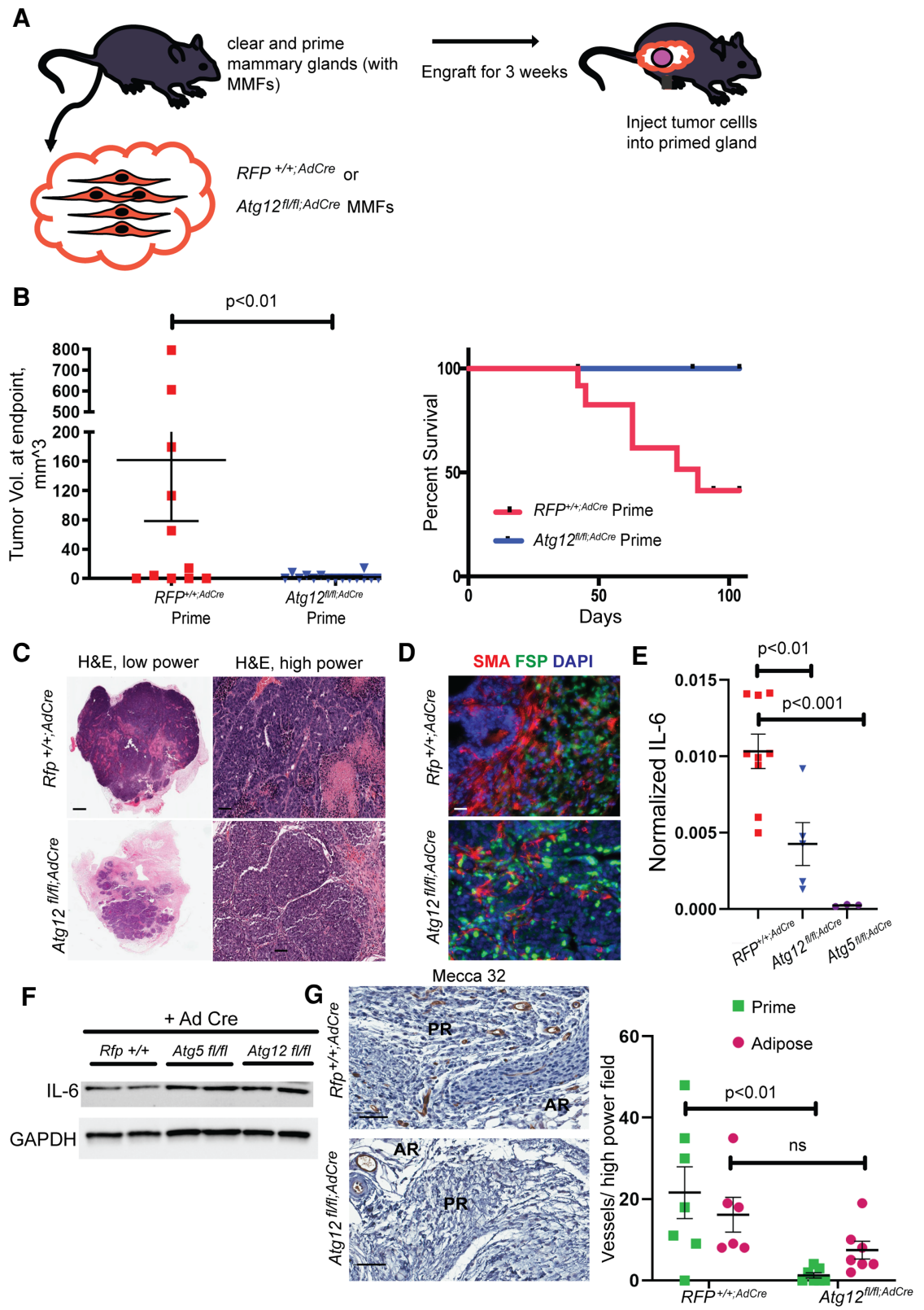

Figure 5. Stromal fibroblast autophagy supports PyMT tumor growth in primed mammary fat pads. (A) Schematic of tumor growth assay in MFPs cleared and primed with autophagy-competent $\left(R F P^{+/+ \text {AdCre }}\right)$ or -deficient (Atg12 1 1/fl;AdCre) MMFs. After 3 wk engraftment, autophagy-competent PyMT tumor cells injected into the primed gland. $(B$, left $)$ Scatter plot showing end point tumor volume (in cubic millimeters) for primed tumors. Data points are individual mice $($ mean \pm SEM) from three independent experiments. End point defined based on mice in the control $\left(R F P^{+/+A d C r e}\right.$ prime $)$ cohort requiring euthanasia. One mouse from $R F P^{+/+; A d C r e}$ cohort with a very large tumor was excluded. $R F P^{+/+ \text {AdCre }}$ prime, $n=12$; Atg12 $2^{f l / f 1 ; A d C r e}$ prime, $n=16$. Mann-Whitney test. (Right) Kaplan-Meier curve showing survival of tumor-burdened mice primed with autophagy-competent $\left(R F P^{+/+; A d C r e}\right.$, $n=12)$ or autophagy-deficient (Atg12 $2^{f l / f 1 ; A d C r e}$, $n=16)$ MMFs after $100 \mathrm{~d}$. (C) H\&E and PyMT immunohistochemistry of tumors grown in fat pads primed with autophagy-competent or -deficient Atg12 $2^{\text {thl; } \text { AdCre }}$ MMFs. (Left panels) Low-power H\&E. (Middle panels) High-power H\&E. (D) Representative immunofluorescence staining of primed tumors for aSMA (red) and FSP (green) expression. Scale bar, $100 \mu \mathrm{m}$. (E) Normalized IL-6 secreted by autophagy-competent $\left(R F P^{+/+; A d C r e}\right)$ or -deficient (Atg12 ${ }^{f l / f 1 ; A d C r e}$ and Atg5 $\left.5^{f l / f l ; A d C r e}\right)$ MMFs. Mean \pm SEM from three independent experiments; $R F P^{+/+; A d C r e}, \quad n=9$; Atg12 $2^{f l / f 1 ; A d C r e}, n=5 ;$ Atg $5^{f l / f l ; A d C r e}, n=3$. One-way ANOVA with Tukey's posttest. $(F)$ Immunoblotting for intracellular IL-6 protein in autophagy-competent $\left(R F P^{+/+ \text {AdCre }}\right)$ or -deficient (Atg5/12 fl/fl;AdCre $)$ MMFs. GADPH used as loading control. (G) Representative Mecca32 immunohistochemistry of autophagy-competent $\left(R F P^{+/+; A d C r e}\right)$ or -deficient $\left(\operatorname{Atg} 12^{f 1 / f 1 ; A d C r e}\right)$ primed MFPs. Scale bar, $50 \mu \mathrm{m}$. (Right) Quantification of microvessel density (MVD) in the fibroblast prime region ( $\mathrm{PR}$; green squares) and the adipose fat pad region (AR; red circles) of primed MFPs.

Mean \pm SEM from two independent experiments; $n=7$ fields from three mice per cohort. Two-way ANOVA, Sidak's multiple comparisons posttest.

MMFs; furthermore, these phenotypes were not significantly enhanced upon Baf A treatment (Fig. 7B-D; Supplemental Fig. S7) in autophagy-deficient cells. These data corroborate that autophagy in mammary fibroblasts supports the lysosomal turnover of procollagen, which contributes to efficient intracellular type 1 procollagen proteostasis in vitro as well as extracellular secretion of type 1 collagen, both in vitro and in vivo. Overall, these results delineate that autophagy in stromal fibroblasts promotes the efficient secretion type 1 collagen, which contributes to the stiff desmoplastic stroma permissive for mammary tumor growth.

\section{Discussion}

Previous reports have shown up-regulation of autophagy in the tumor microenvironment and fibroblasts (Martinez-Outschoorn et al. 2010; Karsli-Uzunbas et al. 2014; Katheder et al. 2017; New et al. 2017) and implicated autophagy in key molecular signaling events involved in protumorigenic paracrine signaling (Narita 2011; Deretic et al. 2013; Lock et al. 2014). To directly interrogate the role of stromal fibroblast autophagy in tumorigenesis, we leveraged genetic loss-of-function approaches to inhibit fibroblast autophagy during PyMT mammary cancer 


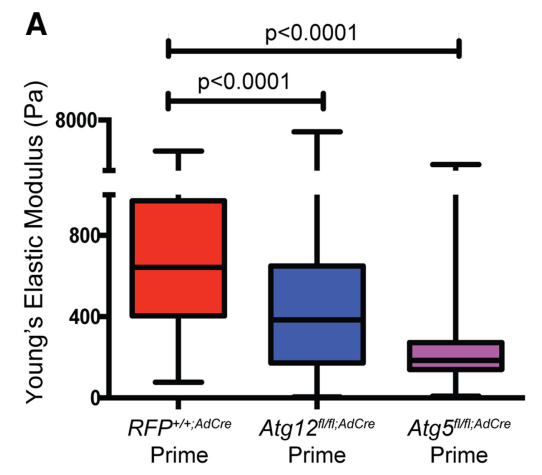

C

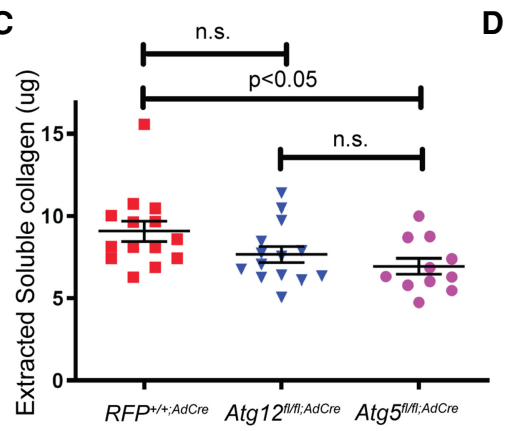

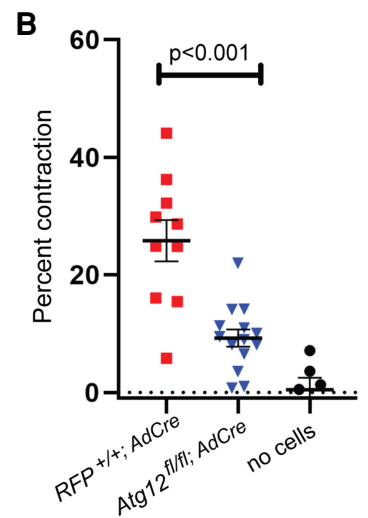

D

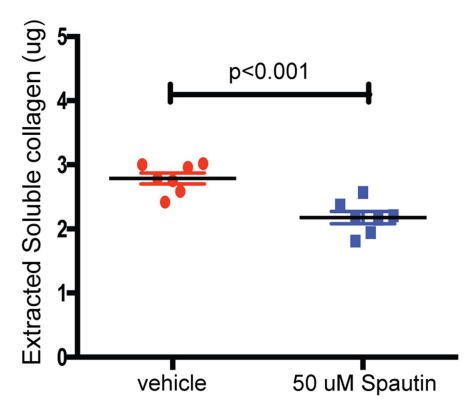

Figure 6. Fibroblast autophagy promotes tissue stiffness and soluble collagen secretion. $(A)$ Box and whiskers plot of Young's elastic modulus $(\mathrm{Pa})$ of primed MFPs using atomic force microscopy. Line indicates median from two independent experiments; whiskers indicate the minimum to maximum. $\mathrm{RFP}^{+/+; A d C r e}$ prime, $n=1724$ measurements from five mice (red); Atg12 fl/fl; AdCre prime (blue), $n=966$ from five mice; Atg $5^{f l / f 1 ; A d C r e}$ prime, $n=685$ from three mice (purple). One-way nonparametric ANOVA, Dunn's multiple comparisons posttest. (B) Average percentage reduction in collagen gel area from FFP $^{+/+; A d C r e}$ or Atg12 $2^{f l / f 1 ; A d C r e}$ MMFs embedded collagen gels. "No cells" condition serves as a negative control for nonspecific gel contraction. Mean \pm SEM from three independent experiments; RFP $^{+/+ \text {AdCre }}, n=10$; Atg12 ${ }^{f l / f ; \text { AdCre }}, n=14$; no cells, $n=6$. Ordinary one-way ANOVA, Tukey's posttest. $(C)$ Extracted soluble collagen secreted by autophagycompetent $\left(R F P^{+/+; A d C r e}\right)$ or -deficient $\left(\right.$ Atg $12^{f l / f f ; A d C r e}$ and Atg $\left.5^{f l / f ; \text { AdCre }}\right)$ MMFs. Mean \pm SEM from four independent experiments. RFP ${ }^{+/+ \text {AdCre }}, \quad n=14$; Atg12 $2^{f l / f l ; d C r e}, n=14 ; A \operatorname{tg} 5^{f l / f ;}$ AdCre,$n=11$. Ordinary one-way ANOVA. $(D)$ Extracted soluble collagen secreted by autophagy-competent $\left(R F P^{+/+; A d C r e}\right)$ MMFs treated with $50 \mu \mathrm{M}$ Spautin-1 for $24 \mathrm{~h}$. Mean \pm SEM from two independent experiments. Vehicle, $n=7$; Spautin, $n=7$. Student's $t$-test. progression. Specifically, we used three different in vivo models: FSP-Cre-mediated deletion of fibroblasts during autochthonous tumor development, cotransplantation of tumor cells with autophagy-deficient or -competent fibroblasts, and MMF priming and tumor cell transplantation. In all these models, our results demonstrate that tumor progression was slowed in the context of autophagy-deficient MMFs. At study end points, tumor volume was also significantly reduced with loss of autophagy in MMFs. The overall consistency between the Atg12 $12^{f l / f 1}$ and $A \operatorname{tg} 5^{f l / f l}$ models in vivo and in vitro supports that phenotypes are due to a general loss of autophagy rather than the individual functions of these proteins. Nevertheless, we do observe certain phenotypic differences between Atg5 and Atg12 ablation in our studies, which may potentially arise from noncanonical functions of ATG5 or ATG12 that do not overlap completely (Galluzzi and Green 2019|. Importantly, in all of these models, autophagy in stromal fibroblasts is required for efficient mammary tumor growth in vivo, even when the cancer cells themselves remain autophagy-competent.

Autophagy deficiency does not globally impair fibrogenic differentiation in mammary stromal fibroblasts. Multiple fibroblast activation markers are intact upon genetic loss of autophagy. Furthermore, examination of the $\mathrm{TGF}^{\beta}$ signaling axis in autophagy-deficient MMFs shows that phospho-SMAD2/3 levels are not reduced, both at baseline and upon exogenous $\mathrm{TGF}^{\beta}$ stimulation in vitro. Rather than broadly impeding fibroblast activation, we propose that autophagy loss elicits specific functional defects in stromal fibroblasts. First, consistent with the requirements for autophagy in energy metabolism, MMFs lacking Atg5 or Atg12 display reduced ATP levels. These reduced ATP levels may contribute to the collagen contraction defect we observed in autophagy-deficient MMFs. More importantly, due to impaired type 1 procollagen proteostasis, autophagy-deficient MMFs exhibit profoundly abrogated collagen secretion in vivo, evidenced by reduced Masson's trichrome and Picrosirius red staining in primed autophagy-deficient mammary fat pads. AFM studies corroborate these defects in collagen deposition elicit significantly reduced matrix stiffness in the stroma generated from autophagy-deficient fibroblasts. Thus, autophagy deficiency in fibroblasts impedes two cardinal features of desmoplastic fibrotic response that promotes tumor growth: increased ECM deposition and tissue stiffness in vivo. In vitro quantification of secreted collagen similarly reveals reduced secretion of soluble collagen in autophagy-deficient MMFs (Forrester et al. 2019).

MMFs lacking autophagy also displayed reduced IL-6 cytokine secretion in vitro consistent with previous reports (Lock et al. 2014; New et al. 2017). However, serum IL-6 levels were intact in adult FSP-Cre;Atg5/12 fl/f1 mice compared with controls. In addition, fibroblast autophagy deficiency did not dramatically inhibit pSTAT3, a marker of IL-6 activation, in either PyMT tumor cells or the associated stroma. Overall, these results argue against impaired IL-6 secretion as a principal contributor to reduced tumorigenesis in the setting of fibroblast autophagy deficiency. Intriguingly, our comixing studies broach the idea that stromal fibroblast autophagy deficiency may actually have tumor-suppressive effects; comixing with autophagy-deficient MMFs significantly reduces tumor volume compared with comixing with either autophagy-competent cells or transplantation of 
A

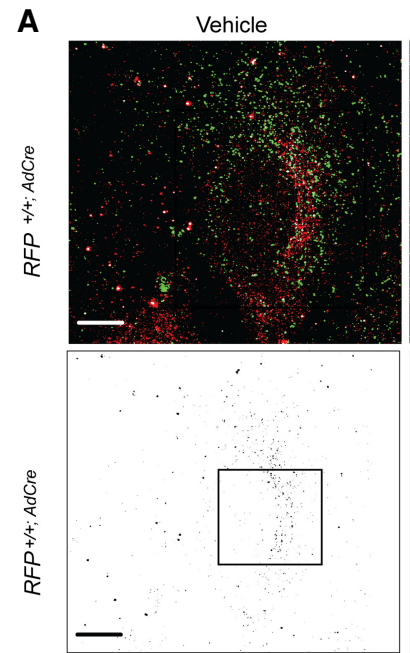

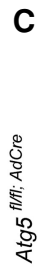
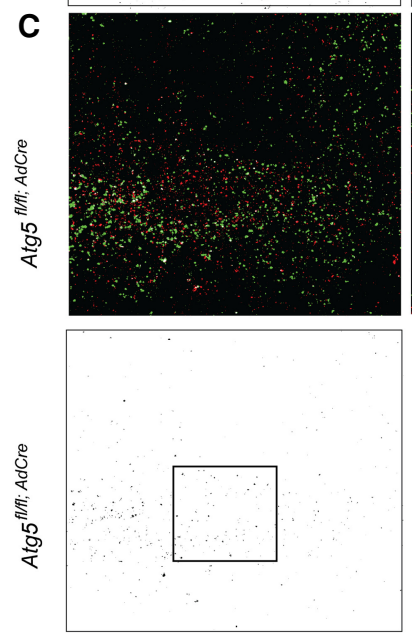
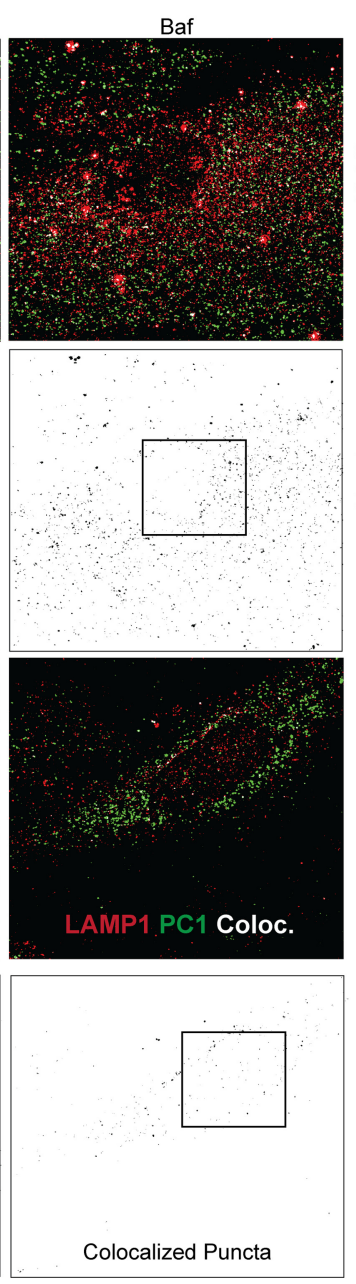

B
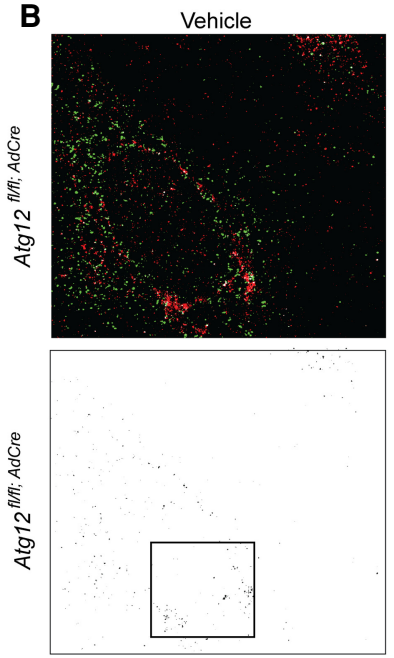

D

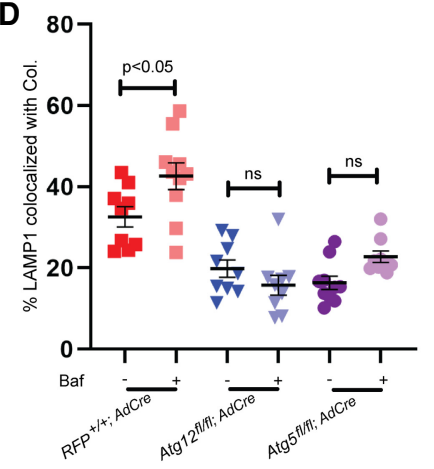

Figure 7. Autophagy-deficient MMFs display impaired lysosomal degradation of type 1 procollagen. Immunofluorescence staining for intracellular type 1 procollagen $\left(\mathrm{PCl}\right.$; green) and lysosomes (LAMP1; red) in $\operatorname{RFP}^{+/+; \text {AdCre }}(A)$, Atg12 fl/fl;AdCre $(B)$, and Atg5 $5^{f l / f 1 ; A d C r e}(C)$ MMFs treated with vehicle (DMSO) or $50 \mathrm{nM}$ bafilomycin A lysosome acidification inhibitor for $12 \mathrm{~h}$. (Top panels) Merged IF images. (Bottom panels) Intracellular colocalized PC1 and LAMP1 (colocalized puncta in black). Boxed areas correspond to enlarged images showing colocalization in Supplemental Figure S7. (D) Quantification of percentage of LAMP1 colocalized with collagen with DMSO or $50 \mathrm{nM}$ Baf A1 for $12 \mathrm{~h}$ in $R F P^{+/+ \text {AdCre }}$ (red squares), Atg12 $2^{\text {fl/f; AdCre }}$ (blue triangles), and Atg $5^{f l / f 1 ; A d C r e}$ (purple circles) MMFs. Unpaired Student's $t$ test. Quantification based on $60 \times$ high-power confocal thresholded images. Scale bar, $10 \mathrm{~nm}$.

PyMT tumor cells only. Further defining these potential tumor suppressive effects remain an important area for future study.

While previous studies have demonstrated various metabolic advantages afforded by autophagy in the host stroma, our results uncover a role for host stromal fibroblast autophagy in supporting the tumor-promoting desmoplastic response, including, most notably, type 1 collagen deposition and tissue stiffness. Furthermore, we reveal that fibroblast autophagy regulates neoangiogenesis and the recruitment of innate and adaptive immune cells. In future work, it will be important to scrutinize whether and how these changes in the vascular and immune microenvironment contribute to tumor progression and desmoplasia. By specifically demonstrating that genetic loss of stromal fibroblast autophagy impedes mammary tumor progression, our results provide funda- mental insight regarding the potentially beneficial impacts of autophagy inhibition in cancer therapy.

Materials and methods

Cell culture

Primary MMFs were grown on tissue culture plastic Petri dishes (Fisher) coated with $5 \mu \mathrm{g} / \mathrm{cm}^{2}$ type 1 rat tail collagen (BD Biosciences) diluted in $0.02 \mathrm{M}$ acetic acid. MMFs were isolated from 6- to

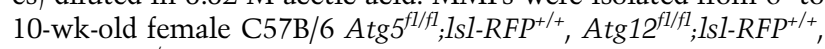
or $1 s l-R F P^{+/+}$mouse mammary glands as previously described (Proia and Kuperwasser 2006). Mammary glands were minced and digested with $125 \mathrm{U} / \mathrm{mL}$ hyaluronidase and $1.5 \mathrm{mg} / \mathrm{mL}$ collagenase (Sigma-Aldrich) in MMF medium (DMEM with $10 \%$ calf serum, $1 \%$ penicillin/streptomycin, $1 \%$ gentamicin) for $\sim 20 \mathrm{~min}$ with vigorous shaking at $37^{\circ} \mathrm{C}$. Collagenase was inactivated by 1 $\mu \mathrm{M}$ EDTA; the mixture was centrifuged at $1000 \mathrm{rpm}$ for $5 \mathrm{~min}$, 
resuspended in MMF medium, and seeded onto collagen-coated 6-cm plates. Fibroblasts from these organoids were maintained for up to three passages. Primary PyMT tumor cells were isolated from female MMTV-PyMT C57Bl/6 mice at 4-5 mo of age and digested as above. Cells were cultured in mammary epithelial cell medium (DMEM/F12 with 10\% fetal bovine serum, 1\% penicillin/streptomycin, $1 \%$ gentamicin, $20 \mathrm{ng} / \mathrm{mL}$ EGF, $0.5 \mu \mathrm{g} / \mathrm{mL}$ hydrocortisone, and $10 \mu \mathrm{g} / \mathrm{mL}$ insulin) on tissue culture-treated 10 $\mathrm{cm}$ plates (Falcon) for up to $3 \mathrm{~d}$ after isolation before use in vivo.

\section{Adenoviral MMF infection}

Adenovirus expressing CMV-driven Cre-recombinase with GFP (Ad5CMVCre-eGFP) $\sim 4 \times 10^{10}$ to $5 \times 10^{10} \mathrm{PFU} / \mathrm{mL}$ was purchased from the University of Iowa Viral Vector Core Facility. MMFs were trypsinized, counted, and infected in suspension overnight in serum-free MMF medium (SF-MMF media, 0.5\% calf serum, $1 \%$ penicillin/streptomycin, $1 \%$ gentamicin) at MOI 480 and then returned to complete MMF media. After $7 \mathrm{~d}$ to allow for autophagy gene ablation, cells were confirmed to be RFP-positive by epifluorescence imaging; lysates were prepared to confirm genetic ATG deletion.

\section{Animal models}

The generation of $A \operatorname{tg} 12^{f l / f 1}$ mice has been described (Malhotra et al. 2015). Atg $5^{f l / f 1}$ mice were obtained from Noboru Mizushima (Kuma et al. 2004). R26-1sl-tdRFP mice were obtained from Hans Joerg Fehling (Luche et al. 2007), bred to $\operatorname{Atg} 12^{f 1 / f 1}$ and $\operatorname{Atg} 5^{f 1 / f 1}$ mice, and backcrossed for more than five generations to $\mathrm{C} 57 \mathrm{~B} / 6$ mice. FSP-Cre (FvB) and MMTV-PyMT (C57/B6) mice were provided by Zena Werb (UCSF). FSP-Cre mice were bred to the $\operatorname{Atg} 12^{f l / f 1}$ and $A \operatorname{tg} 5^{f l / f 1}$ strains and then bred to MMTV-PyMT males to obtain Atg12 ${ }^{f l / f 1} ; 1 s 1-R_{F P} P^{+/} ; P y M T+F S P-C r e+$ and Atg $5^{f l / f 1} ; 1 S 1-R F P^{+/+} ;$PyMT+;FSP-Cre+ mice. All mice were maintained according to UCSF IACUC standards. For tumor studies, mice were monitored for mammary tumor development, and blind measurements were obtained using calipers.

In vivo MFP priming assays

C57Bl/6 mice (22 d old) were purchased from Jackson Laboratories (strain no. 000664). A modified version of the protocol described by Kuperwasser et al. (2004) was used. Mice were anesthetized, and the rudimentary epithelium was cleared from the right inguinal mammary gland. At time of clearing, $5 \times 10^{5}$ MMFs, in a mixture of $25 \%$ Matrigel (BD Biosciences) in MMF media, were injected into the epithelium-devoid fat pad. Three weeks later, $1.6 \times 10^{5}$ primary PyMT cells were diluted in a mixture of $25 \%$ Matrigel and injected into the fibroblast primed right inguinal cleared mammary gland.

\section{In vivo comixing assays}

C57Bl/6 mice (6-8 wk old) were purchased from Jackson Laboratories (strain no. 000664). A mixture of MMFs with primary PyMT tumor cells at a cell ratio of $3: 1$, or PyMT cells only, were diluted in 25\% Matrigel in MMF media and injected into intact fourth inguinal right and left mammary glands.

\section{Fluorescence-activated cell sorting of FSP-Cre mammary glands}

Isolated mammary glands were minced and digested as above. Red blood cells were lysed with red blood cell lysis buffer (Sigma-Aldrich) by manufacturer's instructions. Samples were treated with $100 \mu \mathrm{g} / \mathrm{mL}$ DNase (Sigma-Aldrich) for $2 \mathrm{~min}$ at $37^{\circ} \mathrm{C}$ and filtered using $30-\mu \mathrm{m}$ filters. Cells were counted, resuspended up to $10^{7}$ cells $/ \mathrm{mL}$ per $90 \mu \mathrm{L}$ FACS buffer (PBS $+5 \%$ CS), and treated with FcR blocking reagent (1:10; Miltenyi Biotec 130-092-575) for $30 \mathrm{~min}$ on ice. Cell suspensions were transferred to polypropylene FACS tubes (BD Biosciences) and either left unstained, stained with isotype control (1:50; Biolegend 400511), or stained with CD326-APC (EpCAM, 1:50; Biolegend 118214) for $30 \mathrm{~min}$ on ice. Cells were washed with $1 \mathrm{~mL}$ of FACS buffer and sorted using a Beckman Coulter MoFlo XDP at the UCSF Parnassus Flow Cytometry Core.

\section{Immunoblotting}

Cells were lysed in RIPA buffer (Invitrogen) with protease inhibitor cocktail (Sigma-Aldrich), $10 \mathrm{mM} \mathrm{NaF}, 10 \mathrm{mM} \beta$-glycerophosphate, $0.5 \mathrm{mM}$ PMSF, and $10 \mathrm{nM}$ calyculin A. Lysates were cleared by centrifugation at $12,000 \mathrm{rpm}$ for $10 \mathrm{~min}$ at $4^{\circ} \mathrm{C}$ and stored at $-80^{\circ} \mathrm{C}$. Protein concentration was determined by BCA assay (Pierce). Twenty micrograms of protein was boiled in sample buffer, resolved by SDS-PAGE, and transferred to PVDF membrane at $4{ }^{\circ} \mathrm{C}$. Membranes were blocked for $1 \mathrm{~h}$ in $5 \%$ milk in PBS with $0.1 \%$ Tween 20 (PBST), incubated with primary antibody in $5 \%$ milk/PBST overnight at $4^{\circ} \mathrm{C}$, washed, incubated for $1 \mathrm{~h}$ at room temperature with HRP-conjugated donkey secondary antibodies (Jackson ImmunoResearch) at 1:5000 in 5\% milk/PBST, washed, and developed with Luminol signal reagent (Fisher). Primary antibodies used for immunoblotting were as follows: ATG12 (1:500; Cell Signaling 2011), ATG5 (1:2000; Novus Biologicals NB110-53818), p62 (1:1000; Progen Biotechnik GP62C), GAPDH (1:10,000; EMD Millipore CB1001), tubulin (1:1000; Cell Signaling 2144), IL-6 (1:1000; Abcam 6672), phosphorylated Smad2/3 (1:500; Cell Signaling 8828), Smad2 (1:1000; Cell Signaling 5339), and MAP1LC3 (1:2000; EMD Millipore ABC232). Densitometry was performed with ImageJ/Fiji software.

\section{Immunohistochemistry}

Mammary glands and tumors were fixed in $10 \%$ neutral-buffered formalin and paraffin embedded. Sections $(5-8 \mu \mathrm{m})$ were deparaffinized and rehydrated through xylene and decreasing percentages of ethanol. Sodium citrate antigen retrieval solution at $\mathrm{pH} 6.0$ (Dako) was used for antigen retrieval, except for F4/80 staining, which required $20 \mu \mathrm{g} / \mathrm{mL}$ Proteinase $\mathrm{K}$ (Invitrogen) for $5 \mathrm{~min}$. Endogenous peroxidases were blocked by incubation in $3 \% \mathrm{H}_{2} \mathrm{O}_{2}$ for $20 \mathrm{~min}$, washed, and blocked for $2 \mathrm{~h}$ at room temperature in $1 \%$ BSA supplemented with $10 \%$ goat serum. Primary antibody staining was performed overnight at $4^{\circ} \mathrm{C}$. Biotinylated secondary antibodies (1:400; Vector Laboratories) and the Elite ABC peroxidase kit with DAB substrate or Nova Red substrate kit (Vector Laboratories) were used for detection. Primary antibodies used for immunohistochemistry were as follows: phosphorylated histone H3 (pH 3; 1:100; Cell Signaling 9701), p62 (1:200; Progen Biotechnik GP62-C), aSMA (1:200; Vector Laboratories VP-S281), CK14 (1:1000; Covance PRB-155P), CD45 (1:250; EMD Millipore 05-1416), F4/80 (1:300; AbD Serotec MCA497GA), RFP (1:500; Rockland Immunochemicals 600-401-379), Mecca32 (1:25; Developmental Studies Hybridoma Bank, University of Iowa), CD3 (1:500; DAKO A0452), CD11b (1: 500; Novus Biologicals 110-89474SS), CD4 (TE antigen retrieval; 1: 100; Abcam 183685), and pSTAT3 Tyr705 (1:100; Cell Signaling 9131S).

\section{Immunofluorescence}

For dual immunofluorescence, sections were deparaffinized, rehydrated, antigen retrieved, and blocked as above. The second 
primary antibody was added sequentially before secondary antibody (Alexa) incubation, wash, Hoescht counterstain, and mounting with Prolong Gold (Life Technologies). Primary antibodies used included aSMA (1:200; Vector Laboratories VPS281) and S100A4 (1:50; Abcam 27957). For immunocytochemistry, 15,000 fibroblasts were seeded onto $5 \mu \mathrm{g} / \mathrm{cm}^{2}$ collagen I-coated eight-well chamber slides, fixed in ice-cold methanol for $20 \mathrm{~min}$, air-dried for $5 \mathrm{~min}$, and stored at $-80^{\circ} \mathrm{C}$. Upon thawing, slides were washed in PBS, permeabilized with $0.1 \%$ Triton $\mathrm{X}-100$, blocked, incubated in primary antibody overnight at $4^{\circ} \mathrm{C}$, and detected with Alexa Fluor secondary antibodies. Epifluorescence images were obtained by Axiovert 200 microscope (Carl Zeiss) with a 20× objective and Spot RT camera (Diagnostic Instruments). Primary antibodies used were as follows: aSMA (1:200; Vector Laboratories VP-S281), vimentin (1:100; Novus Biologicals NB100-74564), CK8 (Troma-I; 1:500; Developmental Studies Hybridoma Bank, University of Iowa), CK14 (1:500; Covance PRB-155P), S100A4 (FSP1; 1:50; Abcam 27957), FAP (1:50; Sigma-Aldrich SAB4500839), and fibronectin (1:200; Abcam 2413). Fibroblast activation markers were quantified by measuring average intensity/cell in thresholded images in ImageJ/Fiji.

ATP

The CellTiter-Glo assay (Promega) was used according to the manufacturer's instructions under nutrient-rich conditions. Oligomycin (5 $\mu \mathrm{M}$; Cell Signaling Technologies 9996) was added to some samples, and luminescence evaluated by a Molecular Devices Name SpectraMax M2 plate reader.

\section{Collagen contraction assay}

Type I rat tail collagen (BD Biosciences) was diluted to $3 \mathrm{mg} / \mathrm{mL}$, neutralized with $0.1 \mathrm{M} \mathrm{NaOH}$, and brought to $10 \mathrm{~mL}$ with $10 \times$ $\mathrm{PBS}$ and $\mathrm{dH}_{2} \mathrm{O}$. Infected MMFs were trypsinized and resuspended at $1.2 \times 10^{6}$ cells $/ \mathrm{mL}$ in SF-MMF media (above). A ratio of $200 \mu \mathrm{L}$ of diluted, neutralized collagen was used per $100 \mu \mathrm{L}$ of MMF cell suspension for each technical replicate; $300 \mu \mathrm{L}$ of diluted, neutralized collagen/MMF mixture was seeded into one well of a 24-well plate. Collagen cell lattices were polymerized for $2 \mathrm{~h}$ at $37^{\circ} \mathrm{C}$. Next, $1 \mathrm{~mL}$ of SF-MMF media was added to each well, and the sides of the collagen gel were released by micropipette. The axes of the gel ellipse were measured at "time $0 \mathrm{~h}$ " by Leica dissecting scope and camera. The plates were incubated for $4 \mathrm{~h}$ at $37^{\circ} \mathrm{C}$, and the axes remeasured to calculate percentage reduction in ellipse area.

\section{Secreted collagen}

The Sircol soluble collagen assay (Biocolor) was used following the manufacturer's instructions with overnight incubation. MMFs grown on fibronectin-coated plates were treated with $20 \mu \mathrm{M}$ L-ascorbic acid 2-phosphate (LAA2P; Cayman Chemical) for $24 \mathrm{~h}$, and deposited collagen was acid-extracted and bound to Sircol dye. For Spautin treatment, MMFs were treated at $50 \mu \mathrm{M}$ Spautin-1 (Sigma-Aldrich) for $24 \mathrm{~h}$. Absorbance was measured at $555 \mathrm{~nm}$, and concentration was calculated by standard curve and normalized for DNA content based on 260-nm absorbance.

ELISAs and cytokine arrays

IL-6 (Abcam 100712) and TGF $\beta 1$ (Abcam 119557) mouse-specific ELISAs and mouse-specific cytokine arrays (mouse cytokine array C2000, Ray Biotech AAM-CYT-2000) were performed follow- ing the manufacturer's instructions. CM used for ELISAs or cytokine array was collected by seeding infected MMFs at equal density on collagen-coated plates in full media. The next day, media was changed to SF-MMF medium. After 24-h conditioning, $\mathrm{CM}$ was collected, filtered through a $0.2-\mu \mathrm{m}$ filter, and stored at $-80^{\circ} \mathrm{C}$. Cells were counted for normalization, lysed, and immunoblotted to confirm autophagy deletion. Recombinant mouse $\mathrm{TGF}^{\beta}$ (R\&D Systems) was added at $10 \mathrm{ng} / \mathrm{mL}$ to MMFs plated on collagen for $30 \mathrm{~min}$ in SF media (above) before cell lysis (see Immunoblotting). For serum IL-6, blood was drawn from the inferior vena cava of mice aged $8-12 \mathrm{wk}$ and coagulated for $30 \mathrm{~min}$ at room temperature, and serum was collected by spinning at 2000 rcf for $10 \mathrm{~min}$ at room temperature. IL6 was measured by ELISA (mouse quantikine ELISA, R\&D Biosystems M600B).

\section{Picrosirius red staining}

FFPE sections were stained with Picrosirius red (Direct Red 80, Sigma-Aldrich) and counterstained with Weigert's hematoxylin (Sigma-Aldrich). Imaging was done using polarizers oriented parallel and orthogonal to each other. Collagen was quantified using a set threshold in ImageJ and measuring more than four regions per primed MFP.

\section{H\&E and Masson's trichrome staining}

These stains were performed by the University of California at San Francisco Mouse Pathology Core and scanned with an Aperio ScanScope XT slide scanner (Aperio Technologies) at 40×.

\section{Atomic force microscopy of primed MFPs}

MFPs were OCT-embedded and flash-frozen in liquid nitrogen. Sections were cut at $30 \mu \mathrm{m}$ on Fisher Superfrost Plus gold slides. Immediately prior to AFM, sections were thawed at room temperature, rinsed of OCT in RTPBS, and covered in HEPES-buffered PBS with protease inhibitor cocktail (Roche) and $1 \mu \mathrm{g} / \mathrm{mL}$ propidium iodide (Invitrogen). Slides were magnet-anchored to a Nikon TE2000-U inverted fluorescent microscope equipped with an Asylum Research MFP-3D atomic force microscope as previously described (Lopez et al. 2011). All samples were measured in liquid media in contact mode using Novascan cantilevers $(2.5-\mu \mathrm{m}$ radius beaded silica glass tip, $\mathrm{k}=0.06 \mathrm{~N} / \mathrm{m})$, calibrated by the thermal tune method. Force measurements were collected over a $90-\mu \mathrm{m} \times 90$ - $\mu \mathrm{m}$ grid at a resolution of $16 \mu \mathrm{m} \times 16 \mu \mathrm{m}$ at a trigger force of $4 \mathrm{nN}$. The force data were converted to elastic modulus using the Hertz Model program (tissue samples assumed to be incompressible and a Poisson's ratio of 0.5 was used to calculate Young's elastic modulus) in IgorPro v6.22, supplied by Asylum Research.

Bafilomycin treatment and immunocytochemistry for type 1 procollagen

Fifty thousand cells were plated on glass coverslips coated with fibronectin 1 (Corning) at $50 \mu \mathrm{g} / \mathrm{mL}$ in PBS for $1 \mathrm{~h}$ at $37^{\circ} \mathrm{C}$, rinsed with PBS, and treated $\sim 24$ h later. Bafilomycin or DMSO (Sigma) was added to MMF media for $12 \mathrm{~h}$. Cells were fixed in 4\% PFA in dPBS for 20 min at $4{ }^{\circ} \mathrm{C}$, permeabilized in $0.5 \%$ Triton $\mathrm{X}$ in PBS for $5 \mathrm{~min}$ at $4^{\circ} \mathrm{C}$, autofluorescence-quenched in $100 \mathrm{mM}$ PBS-glycine wash, and blocked in PBS with $10 \%$ goat serum and $1 \%$ BSA. Cells were stained using anti-LAMP1 (1:2000; BD Pharmingen 1D4B) and type 1 procollagen ( $\mathrm{PC1} ; 1: 100 ;$ LF42) overnight at $4^{\circ} \mathrm{C}$, and Alexa Fluor secondary antibodies were used (1:1000 and 1:500, respectively). Hoechst (Invitrogen) was used at 0.002 $\mathrm{mg} / \mathrm{mL}$ in $\mathrm{dPBS}$ for $12 \mathrm{~min}$ at room temperature, and slides 
were mounted in VectaShield mounting media (Vector Laboratories). Slides were imaged at $60 \times$ with a confocal DeltaVision Elite (General Electric). The number of $\mathrm{PC1}$ particles colocalized with LAMP1 was analyzed using thresholded images in Fiji. Each data point represents one $60 \times$ image with more than two cells. Similar staining protocols were used for Ki67 immunocytochemistry (1:200; Abcam 15580).

\section{Quantitative RT-PCR}

RNA was extracted by RNeasy kit (Qiagen), reverse-transcribed, and PCR-amplified using the StepOnePlus system (Applied Biosystems). Primers used were as follows: colla1 (forward, GCTC CTCTTAGGGGCCACT; reverse, CCACGTCTCACCATTGG GG), lox (forward, GCCGACCAAGATATTCCTGGG; reverse, GCAGGTCATAGTGGCTAAACT), loxl2 (forward, GGTGGAG GTGTACTATGATGG; reverse, CTTGCCGTAGGAGGAG CT), tgf $\beta 1$ (forward, CTCCCGTGGCTTCTAGTGC; reverse, GCCTTAGTTTGGACAGGATCTG), and rpl7 to normalize gene expression (forward, ACCGCACTGAGATTCGGATG; reverse, GAACCTTACGAACCTTTGGGC).

Statistical analyses

GraphPad Prism software (v6.0) was used for graphs and statistics. To generate $P$-values for Gaussian distributed data groups of three of more, a one- or two-way ordinary ANOVA was used with Tukey's posttest or Sidak's multiple comparisons test, respectively. For pairwise comparisons of Gaussian distributed data, a Student's $t$-test was used. For nonparametric distributed data groups of three or more, a nonparametric ANOVA and Dunn's multiple comparisons test was used. For pairwise comparisons of nonparametric distributed data, a Mann-Whitney test was used. For statistical comparisons, the variance between groups was similar.

\section{Competing interest statement}

J.D. is a scientific advisory board member of Vescor Therapeutics, LLC, and a consultant for Merck, Inc.

\section{Acknowledgments}

We thank Noboru Mizushima (University of Tokyo) and Hans Joerg Fehling (Ulm University) for generously providing mice and Dr. Larry Fischer (National Institutes of Health $[\mathrm{NIH}]$ ) for the procollagen antibody. Fluorescence-activated cell sorting was performed by Ninnia Lescano (University of California at San Francisco [UCSF] Parnassus Flow Cytometry Core). Tissue sectioning was performed by Jennifer Bolen (UCSF Mouse Pathology Core). Atomic force microscopy was assisted by Luke Cassereau (UCSF). Grant support to J.D. includes the NIH (CA126792, CA188404, and CA201849), Department of Defense Breast Cancer Research Program (DOD GCRP; W81XWH-11-1-0130 and W81XWH-12-1-0505), Samuel Waxman Cancer Research Foundation, and Mark Foundation for Cancer Research (Endeavor Award). J.A.R. was supported by the DOD BCRP postdoctoral award (W81XWH-13-1-0217), and J.M.B. was supported by NIH Postdoctoral Fellowship F32CA174319. F.A.M., H.S., and J.G. are supported by National Science Foundation graduate student fellowships (DGE-1144247). T.M. is supported by Institutional Research and Academic Career Development Awards K12GM081266 and American College of Surgeons/Jean Perkins Foundation fellowship PF-18-227-01-CSM.
Author contributions: J.A.R. and J.D. originated the study, which was completed by T.M., including experiments for revisions. J.A.R., T.M., and J.D. designed experiments with input from J.M.B. and V.M.W. for collagen analysis and tissue stiffness. J.A.R., T.M., F.A.M., J.M.B., H.S., J.G., S.R., and S.B.E. performed experiments and analyzed the data. J.A.R., T.M., and J.D. wrote the manuscript, with input from all authors.

\section{References}

Amaravadi RK, Kimmelman AC, Debnath J. 2019. Targeting autophagy in cancer: recent advances and future directions. Cancer Discov 9: 1167-1181. doi:10.1158/2159-8290.CD-190292

Bhowmick NA, Chytil A, Plieth D, Gorska AE, Dumont N, Shappell S, Washington MK, Neilson EG, Moses HL. 2004. TGF- $\beta$ signaling in fibroblasts modulates the oncogenic potential of adjacent epithelia. Science 303: 848-851. doi:10.1126/sci ence.1090922

Bromberg J, Wang TC. 2009. Inflammation and cancer: IL-6 and STAT3 complete the link. Cancer Cell 15: 79-80. doi:10 .1016/j.ccr.2009.01.009

Bunt SK, Yang L, Sinha P, Clements VK, Leips J, Ostrand-Rosenberg S. 2007. Reduced inflammation in the tumor microenvironment delays the accumulation of myeloid-derived suppressor cells and limits tumor progression. Cancer Res 67: 10019-10026. doi:10.1158/0008-5472.CAN-07-2354

Cadwell K, Debnath J. 2018. Beyond self-eating: the control of nonautophagic functions and signaling pathways by autophagyrelated proteins. J Cell Biol 217: 813-822. doi:10.1083/jcb .201706157

Cinque L, Forrester A, Bartolomeo R, Svelto M, Venditti R, Montefusco S, Polishchuk E, Nusco E, Rossi A, Medina DL, et al. 2015. FGF signalling regulates bone growth through autophagy. Nature 528: 272-275. doi:10.1038/nature16063

Deome KB, Faulkin LJ Jr, Bern HA, Blair PB. 1959. Development of mammary tumors from hyperplastic alveolar nodules transplanted into gland-free mammary fat pads of female $\mathrm{C} 3 \mathrm{H}$ mice. Cancer Res 19: 515.

Deretic V, Jiang S, Dupont N. 2013. Autophagy intersections with conventional and unconventional secretion in tissue development, remodeling and inflammation. Trends Cell Biol 22: 397-406. doi:10.1016/j.tcb.2012.04.008

Erez N, Truitt M, Olson P, Hanahan D. 2010. Cancer-associated fibroblasts are activated in incipient neoplasia to orchestrate tumor-promoting inflammation in an NF-kB-dependent manner. Cancer Cell 17: 135-147. doi:10.1016/j.ccr.2009.12.041

Fielding CA, Jones GW, McLoughlin RM, McLeod L, Hammond VI, Uceda J, Williams AS, Lambie M, Foster TL, Liao CT, et al. 2014. Interleukin-6 signaling drives fibrosis in unresolved inflammation. Immunity 40: 40-50. doi:10.1016/j .immuni.2013.10.022

Fischer KR, Durrans A, Lee S, Sheng J, Li F, Wong S, Choi H, Rayes TE, Ryu S, Schwabe RF, et al. 2015. EMT is not required for lung metastasis but contributes to chemoresistance. Nature 527: 472-476. doi:10.1038/nature 15748

Forrester A, De Leonibus C, Grumati P, Fasana E, Piemontese M, Staiano L, Fregno I, Raimondi A, Marazza A, Bruno G, et al. 2019. A selective ER-phagy exerts procollagen quality control via a Calnexin-FAM 134B complex. EMBO J 38: 1-16. doi:10 $.15252 / \mathrm{embj} .201899847$

Galluzzi L, Green DR. 2019. Autophagy-independent functions of the autophagy machinery. Cell 177: 1682-1699. doi:10 .1016/j.cell.2019.05.026 
Hernández-Gea V, Ghiassi-Nejad Z, Rozenfeld R, Gordon R, Fiel MI, Yue Z, Czaja MJ, Friedman SL. 2012. Autophagy releases lipid that promotes fibrogenesis by activated hepatic stellate cells in mice and in human tissues. Gastroenterology 142: 938-946. doi:10.1053/j.gastro.2011.12.044

Karsli-Uzunbas G, Guo JY, Price S, Teng X, Laddha SV, Khor S, Kalaany NY, Jacks T, Chan CS, Rabinowitz JD, et al. 2014. Autophagy is required for glucose homeostasis and lung tumor maintenance. Cancer Discov 4: 914-927. doi:10.1158/ 2159-8290.CD-14-0363

Katheder NS, Khezri R, O'Farrell F, Schultz SW, Jain A, Schink KO, Theodossiou TA, Johansen T, Juhász G, Bilder D, et al. 2017. Microenvironmental autophagy promotes tumour growth. Nature 541: 417-420. doi:10.1038/nature20815

Kojima Y, Acar A, Eaton EN, Mellody KT, Scheel C, Ben-Porath I, Onder TT, Wang ZC, Richardson AL, Weinberg RA, et al. 2010. Autocrine TGF- $\beta$ and stromal cell-derived factor- 1 (SDF-1) signaling drives the evolution of tumor-promoting mammary stromal myofibroblasts. Proc Natl Acad Sci 107: 20009-20014. doi:10.1073/pnas.1013805107

Kuma A, Hatano M, Matsui M, Yamamoto A, Nakaya H, Yoshimori T, Ohsumi Y, Tokuhisa T, Mizushima N. 2004. The role of autophagy during the early neonatal starvation period. $\mathrm{Na}$ ture 432: 1032-1036. doi:10.1038/nature03029

Kuperwasser C, Chavarria T, Wu M, Magrane G, Gray JW, Carey L, Richardson A, Weinberg RA. 2004. Reconstruction of functionally normal and malignant human breast tissues in mice. Proc Natl Acad Sci 101: 4966-4971. doi:10.1073/pnas .0401064101

LeBleu VS, Kalluri R. 2018. A peek into cancer-associated fibroblasts: origins, functions and translational impact. DMM Dis Model Mech 11: dmm029447.

Liao Z, Dai Z, Cai C, Zhang X, Li A, Zhang H, Yan Y, Lin W, Wu $\mathrm{Y}, \mathrm{Li} \mathrm{H}$, et al. 2019. Knockout of Atg5 inhibits proliferation and promotes apoptosis of DF-1 cells. Vitr Cell Dev Biol Anim 55: 341-348. doi:10.1007/s11626-019-00342-7

Lin EY, Jones JG, Li P, Zhu L, Whitney KD, Muller WJ, Pollard JW. 2003. Progression to malignancy in the polyoma middle $T$ oncoprotein mouse breast cancer model provides a reliable model for human diseases. Am I Pathol 163: 2113-2126. doi:10.1016/S0002-9440(10)63568-7

Lock R, Kenific CM, Leidal AM, Salas E, Debnath J. 2014. Autophagy-dependent production of secreted factors facilitates oncogenic RAS-driven invasion. Cancer Discov 4: 466479. doi:10.1158/2159-8290.CD-13-0841

Lopez JI, Kang I, You WK, McDonald DM, Weaver VM. 2011. In situ force mapping of mammary gland transformation. Integr Biol 3: 910-921. doi:10.1039/clib00043h

Luche H, Weber O, Rao TN, Blum C, Fehling HJ. 2007. Faithful activation of an extra-bright red fluorescent protein in 'knock-in' Cre-reporter mice ideally suited for lineage tracing studies. Eur J Immunol 37: 43-53. doi:10.1002/eji.200636745

Malhotra R, Warne JP, Salas E, Xu AW, Debnath J. 2015. Loss of Atg12, but not Atg5, in pro-opiomelanocortin neurons exacerbates diet-induced obesity. Autophagy 11: 145-154.

Martinez-Outschoorn UE, Trimmer C, Lin Z, Whitaker-Menezes D, Chiavarina B, Zhou J, Wang C, Pavlides S, Martinez-Cantarin MP, Capozza F, et al. 2010. Autophagy in cancer associated fibroblasts promotes tumor cell survival: role of hypoxia, HIF1 induction and NFKB activation in the tumor stromal microenvironment. Cell Cycle 9: 3515-3533. doi:10.4161/cc.9 .17 .12928

Monkkonen T, Debnath J. 2018. Inflammatory signaling cascades and autophagy in cancer. Autophagy 14: 190-198. doi:10 $.1080 / 15548627.2017 .1345412$

Narita M. 2011. Spatial coupling of mTOR. Autophagy 966: $966-$ 970.

New J, Arnold L, Ananth M, Alvi S, Thornton M, Werner L, Tawfik O, Dai H, Shnayder Y, Kakarala K, et al. 2017. Secretory autophagy in cancer-associated fibroblasts promotes head and neck cancer progression and offers a novel therapeutic target. Cancer Res 77: 6679-6691. doi:10.1158/0008-5472.CAN17-1077

Poillet-Perez L, Xie X, Zhan L, Yang Y, Sharp DW, Hu ZS, Su X, Maganti A, Jiang C, Lu W, et al. 2018. Autophagy maintains tumour growth through circulating arginine. Nature 563: 569-573. doi:10.1038/s41586-018-0697-7

Proia DA, Kuperwasser C. 2006. Reconstruction of human mammary tissues in a mouse model. Nat Protoc 1: 206-214. doi:10 $.1038 /$ nprot.2006.31

Rønnov-Jessen L, Petersen OW. 1993. Induction of $\alpha$-smooth muscle actin by transforming growth factor- $\beta 1$ in quiescent human breast gland fibroblasts. Implications for myofibroblast generation in breast neoplasia. Lab Invest 68: 696-707.

Rudnick JA, Kuperwasser C. 2012. Stromal biomarkers in breast cancer development and progression. Clin Exp Metastasis 29: 663-672. doi:10.1007/s10585-012-9499-8

Schedin P, Keely PJ. 2011. Mammary gland ECM remodeling, stiffness, and mechanosignaling in normal development and tumor progression. Cold Spring Harb Perspect Biol 3: a003228-. doi:10.1101/cshperspect.a003228

Sousa CM, Biancur DE, Wang X, Halbrook CJ, Sherman MH, Zhang L, Kremer D, Hwang RF, Witkiewicz AK, Ying H, et al. 2016. Pancreatic stellate cells support tumour metabolism through autophagic alanine secretion. Nature 536: 479483. doi:10.1038/nature 19084

Thoen LFR, Guimarães ELM, Dollé L, Mannaerts I, Najimi M, Sokal E, Van Grunsven LA. 2011. A role for autophagy during hepatic stellate cell activation. I Hepatol 55: 1353-1360. doi:10.1016/j.jhep.2011.07.010 


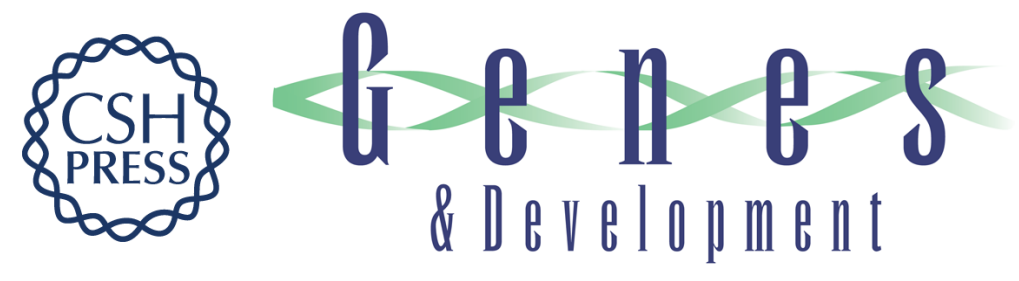

\section{Autophagy in stromal fibroblasts promotes tumor desmoplasia and mammary tumorigenesis}

Jenny A. Rudnick, Teresa Monkkonen, Florie A. Mar, et al.

Genes Dev. 2021, 35: originally published online June 24, 2021

Access the most recent version at doi:10.1101/gad.345629.120

\section{Supplemental http://genesdev.cshlp.org/content/suppl/2021/06/22/gad.345629.120.DC1 Material}

References This article cites 37 articles, 11 of which can be accessed free at: http://genesdev.cshlp.org/content/35/13-14/963.full.html\#ref-list-1

Creative This article is distributed exclusively by Cold Spring Harbor Laboratory Press for the first Commons six months after the full-issue publication date (see

License http://genesdev.cshlp.org/site/misc/terms.xhtml). After six months, it is available under a Creative Commons License (Attribution-NonCommercial 4.0 International), as described at http://creativecommons.org/licenses/by-nc/4.0/.

Email Alerting Receive free email alerts when new articles cite this article - sign up in the box at the top Service right corner of the article or click here.

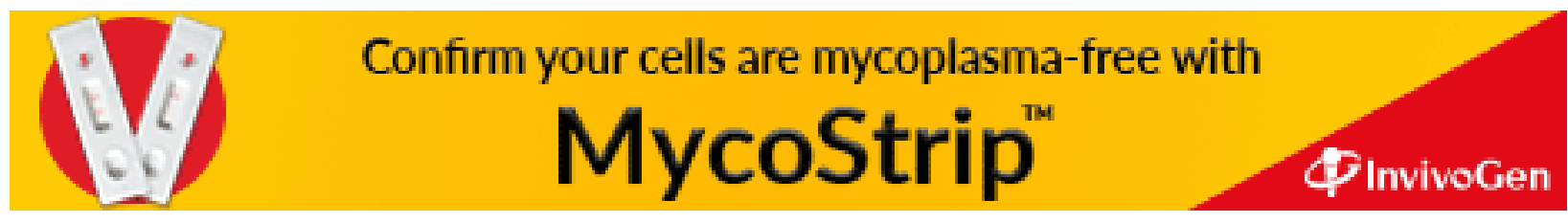

